\title{
Implication of planned radiotherapy on breast reconstruction: radiotherapy and plastic surgery with implants
}

\author{
G. M. Freedman \\ Department of Radiation Oncology, Fox Chase Cancer Center, Philadelphia, PA, USA.
}

\begin{abstract}
Radiation therapy is an important part of the modern multimodality treatment of breast cancer, and in the past decade has had an increasing role in the treatment of patients at intermediate to high risk for localregional recurrence after mastectomy. During the same time period, a less radical surgical approach to mastectomy has developed that is more compatible with reconstruction, and a greater number of young patients diagnosed due to screening who are more motivated and candidates for the procedure. This has led to an increased need to consider the special implications of combining radiation with breast reconstruction. This review will focus on reconstruction by tissue expander and breast implants. A multidisciplinary approach to management is needed that includes a team of a plastic surgeon, and radiation, surgical and medical oncologists. Experience with implant reconstruction and radiation in the past decade has been associated with relatively high rates of complications and/or implant loss compared with unirradiated patients. However, newer techniques of radiation, and improved patient selection and coordination between the multidisciplinary team offer the promise of low complication rates and good or excellent cosmetic results for most patients in the future.
\end{abstract}

Keywords: Breast cancer; Breast implants; Breast reconstruction; Postmastectomy radiation; Radiation therapy

\section{Introduction}

This review focuses on the special challenges posed by the combination of breast reconstruction with implants and radiation therapy. The subject is difficult to analyze from an evidence-based approach because the literature remains small compared to the larger body of literature on postmastectomy radiation in general, and it is limited to single-institution retrospective reviews with relatively few patients. In addition, the case series often are made up of a diverse mix of patients treated with various implant and radiation

Correspondence to: Gary M. Freedman, MD, Department of Radiation Oncology, Fox Chase Cancer Center, 333, Cottman Avenue, Philadelphia, PA 19111, USA. E-mail: G_Freedman@FCCC.edu; Tel: +1 215728 3016; Fax: +12152141629

Publication date 31/08/05

BCO/378/2005/FO techniques, and different combinations of sequencing the reconstruction with mastectomy and radiation. If there can be one conclusion from the published experience, it is that a multidisciplinary approach to patient management is required. There are special considerations for the surgical oncologist and plastic surgeon that include patient selection, timing of implant reconstruction, and choosing a technique of one- or two-stage implant procedure. Coordination of implant expansion and managing postoperative complications is needed between the plastic surgeon and medical oncologist for patients requiring adjuvant chemotherapy. Once postmastectomy radiation has been recommended, there are also special technical considerations for the treating radiation oncologist. And for the entire oncologic team, there is a need for close monitoring and an awareness of the patient problems and complications unique to implants that may occur. 


\section{Background}

Radiation therapy is an integral part of the multimodality treatment of breast cancer. While it has enjoyed a well-established role after breast-conserving surgery, for over 40 years radiation therapy also has been used after mastectomy to the chest wall with or without the regional nodes to reduce local-regional recurrence, but with various degrees of success in improving survival [1-3]. In recent years, there has been increasing evidence from prospective randomized trials and large meta-analyses supporting greater utilization of radiation therapy for patients at high risk for local-regional relapse after mastectomy [4-8]. Current controversy is now more focused on defining the role of radiation for intermediate risk patient subgroups, issues of sequencing with other therapies, and the importance of regional lymph node treatment. Today, there is broad consensus on indications for postmastectomy radiation that make approximately one-third of patients eligible for treatment after mastectomy [9].

With an increase in indications for postmastectomy radiation, there will be an increased need to consider the special implications of combining radiation with breast reconstruction. More women today have the option for breast reconstruction because of advances in surgical techniques. Implants now come in a variety of forms including expander prostheses, with or without detachable valves for one- and twostage procedures. The move to less radical mastectomy that spares the pectoralis fascia, and acceptance of skin-sparing mastectomy, has increased the number of women eligible for implant reconstruction. Older fears of a negative impact on recurrence risk or cancer detection after breast reconstruction have been disproved. And patients diagnosed at a younger age caused by the increased prevalence of mammogram screening may be healthier and more inclined to be eligible for and motivated for breast reconstruction. While there has been considerable progress in the past decade in developing pedicle and free tissue transfer to provide options for women not candidates for or not desiring implants, this will not be discussed in this article.

\section{Immediate vs. delayed reconstruction}

Once a decision has been made between a patient and the plastic surgeon for breast reconstruction by an implant, the next decision to be made is regarding the option for either immediate or delayed reconstruction.

In clinical practice, reconstruction is often delayed after mastectomy until after completion of all adjuvant chemotherapy or radiation therapy. Some authors have gone further and recommended against immediate reconstruction in the case of patients known to require postmastectomy radiation $[10,11]$. For the radiation oncologist, treatment of the unreconstructed chest wall is less technically challenging than a reconstructed breast. Radiation may be done with traditional techniques using widely available conventional simulation and therapy machines. Regional node treatment if indicated may be done with standard matching techniques, without concern for special problems or risks caused by matching fields adjacent to or through an implant. And for the treating radiation oncologist there has been good reason to fear higher risks of complications and poor cosmetic results caused by early reports of radiation therapy with implants [12-18]. Other reasons for delaying the implant may include patient preference for that timing, or a delay in the patient coming to the decision about reconstruction. Also, while most patients should be able to begin chemotherapy within the usual 3-4 weeks after surgery and reconstruction, in the event of a serious infection or delayed wound healing there could be a delay in systemic therapy. This fear may be greater for the patient with locally advanced breast cancer. This concern for delay in chemotherapy or performing reconstruction in those with a high risk of recurrence could cause some to recommend an arbitrary period of time such as 2 years until it is considered 'safe' to proceed with reconstruction, similar to usual warnings about pregnancy within 2 years of treatment that lack supporting evidence.

However, delaying reconstruction with an implant until after radiation therapy is completed may be impossible, or at least more difficult. Full expansion of a tissue expander may not be possible after a patient has undergone radiation therapy due to fibrosis and decreased elasticity of the skin. Dickson and Sharpe [19] reported $50 \%$ (5 of 10) patients with difficult or failed expansion after radiation. Kraemer et al. [17] reported more painful expansion, less over-expansion, worse cosmetic results and more capsulotomies in patients having reconstruction after radiation. Forman et al. [20] reported a $60 \%$ (6 of 10) rate of implant complications after two-stage reconstruction following salvage mastectomy with a history of prior radiation therapy. Their implant complications included difficult expansion, infection, and severe contracture. And recently Tallet et al. [21] reported a complication rate of $63 \%$ (5 of 8 ) in patients undergoing tissue expansion after history of breast-conserving surgery and radiation. Autologous muscle transfer is preferred for patients with a prior history of chest wall radiation, but this may not be possible for many patients due to factors such as prior surgery, lack of sufficient tissue, smoking history, or other comorbidity. In cases where an implant is required to reconstruct an irradiated chest wall, then the simultaneous use of muscle transfer to 
improve coverage of the implant such as a latissimus flap is recommended.

Any policy of selecting delayed reconstruction for patients with preoperative indications for postmastectomy radiation, and immediate reconstruction for those without, will not be an effective means of preventing radiation of implants. It is usually not known with certainty whether a patient will require postmastectomy radiation therapy prior to mastectomy. Definite indications for patients requiring radiation are tumor size $>5 \mathrm{~cm}$, four or more positive lymph nodes, or deep fascia invasion $[2,7,9,22]$. There are also a number of relative indications for radiation such as 1-3 positive nodes, small number of nodes dissected, close or positive margins, or lymphovascular invasion; alone or in combinations these factors may place a patient at intermediate risk of localregional recurrence $[4-8,22,23]$. All of these factors are not usually known until after surgery.

Since many patients offered immediate reconstruction nonetheless postoperatively are found to have indications for radiation therapy, patients having immediate reconstruction may be treated by a delayed two-stage implant reconstruction technique. This 'sandwich' technique involves placing a tissue expander as usual at time of mastectomy, but delaying the completion with a permanent implant after expansion until after radiation. This has many potential advantages with regards to radiation. The expansion of the initial implant is done prior to radiation when the skin, subcutaneous tissue and pectoralis muscle are fully pliable without radiation fibrosis. The temporary tissue expander is irradiated, and then an unirradiated permanent implant exchanged after a delay of a few months or longer if needed for the acute sequelae of radiation to subside. Also, at the time of permanent implant placement, a capsulectomy may be performed if needed due to the early development of radiation fibrosis. This two-stage approach may be associated with a lower long-term rate of complications or re-operation because of fibrosis and contracture.

Lastly, and perhaps most importantly, immediate reconstruction in general is associated with many advantages to the patient compared with delayed reconstruction. Immediate timing during the mastectomy will provide the patient with an important cosmetic and psychological benefit, not awaking from mastectomy with a complete absence of a breast. Delaying reconstruction until after completion of all adjuvant chemotherapy and radiation may translate into a patient waiting 6-9 months for the procedure. Immediate reconstruction is also associated with avoidance of a second operation with its associated risks including anesthesia and infection and other perioperative complications. The inconvenience and cost of a second hospitalization is also avoided. For these reasons, immediate timing of reconstruction with mastectomy should remain an option and may be preferable for most patients choosing or requiring reconstruction by tissue expansion/saline implant.

\section{Radiation therapy techniques}

Conventional photon beam irradiation is widely available and most commonly used for postmastectomy radiation, although electron beam radiation has been used as well [24]. The target volume for radiation is the subcutaneous tissue and lymphatics of the chest wall that could also include any potential retained breast tissue after mastectomy. In patients treated without radiation with implants placed in a submuscular location, the recurrences occur almost always in the subcutaneous tissue anterior to the pectoral muscle and implant. Skin recurrences are infrequent except in cases of inflammatory breast cancer. The target volume should include the entire chest wall to a recommended dose of 5000 cGy (200 cGy per fraction) or 5040 cGy (180 cGy per fraction) [13,21,24-28]. A scar boost is often employed in the postmastectomy setting to increase dose $[13,21,25,26]$. However, because there is not a direct correlation between the tumor location and the placement of the mastectomy scar, the utility of the scar boost remains unproven [29]. This is also true of the locations of close or positive pathologic margins, which cannot be accurately pinpointed for focal boosting after mastectomy as is possible after a lumpectomy. Given the increased doses involved, the boost should be avoided to minimize the risk of complications particularly in the setting of the reconstructed breast.

In patients whom a decision is made to treat the internal mammary nodes, shallow photon fields covering the majority of the central and lateral chest wall can be matched to an angled shallow electron field covering both the medial chest wall and internal mammary nodes [30]. The internal mammary field should be individualized to each patient's anatomy with computed tomography (CT)-based planning. This will help optimization of the technique that treats to an appropriate depth for nodal coverage, and allows three-dimensional dosimetry of the dose homogeneity and dose to the normal tissue and reconstruction. The technique involves feathering the matchline of an anterior electron beam and shallow tangents $0.5-1 \mathrm{~cm}$ each day during treatment. The advantages of this technique over others are that it spreads out the skin dose and minimizes the potential for fibrosis along the matchline of the beams on the skin or through the implant, which may lead to poor cosmetic result or contracture. It also reduces any under-dosed or 'cold' tissue under the matchline of photon and electron 
fields, and minimizes dose given to and volume of underlying heart or lung tissue irradiated.

The placement of a tissue-equivalent bolus material over the chest wall is recommended to account for differences in tissue contour over the reconstructed breast and ensure full dose to the subcutaneous tissue [24-28]. This is usually applied every other day during radiation therapy. Bolus has in the past been associated with a worse cosmetic outcome $[26,27]$. For example, Victor et al. [26] reported a good to excellent cosmetic result in $87 \%$ without bolus and $37 \%$ with bolus $(P=0.016)$. More recently, Anderson et al. [25] have reported on the use of a customfashioned bolus for the reconstructed breast. This is an inexpensive custom-shaped wax cast of the patient's chest that improves upon the bolus material's conformity to the reconstructed breast. The custom bolus reduces air gaps or other variations in thickness seen with standard flat sheets of bolus material. The thickness and conformity of the bolus to the patient's contour can be checked on each patient during a CT-based simulation. The effect of the bolus can also be verified by direct measurements of the skin dose during the first week of radiation at different positions over the reconstructed breast. This technique was associated with a reduced risk of complications compared with their previous use of a standard $1 \mathrm{~cm}$ bolus ( $9 \%$ vs. $24 \%, P=0.05)$, and very high rates of good or excellent cosmetic results.

Intensity modulated radiation therapy (IMRT) represents a more advanced form of 3-D conformal radiation therapy [31]. Conventional chest wall irradiation using standard tangential radiation techniques is often associated with significant dose heterogeneity greater than $10 \%$ increases in dose given to portions of the breast and skin, which also may be true for an implant and reconstructed breast. This dose heterogeneity may negatively impact upon the acute side effects and long-term cosmesis from radiation [32,33]. Higher doses to the skin than intended could increase the risk of desquamation placing the implant at higher risk for infection or exposure. The dose heterogeneity in the intact breast setting also has been associated with worse long-term cosmetic outcome and complications such as fibrosis or skin telangiectasias [34-36]. Dose heterogeneity may be reduced with conventional radiation by means of higher energy beams with beam spoilers or custom-fashioned bolus. With IMRT, the intensity of the beam itself is varied across the aperture to shape dose distributions around targets in a way previously not possible with conventional techniques. Initial experiences with IMRT after breast-conserving surgery have been shown clinical feasibility, improved dose distributions in the treated breast, lower doses given to normal heart or lung tissue compared with standard techniques, a low incidence of acute skin toxicity, and excellent cosmetic results at 1 year [37-41]. In our early clinical experience in 73 women with early stage breast cancer treated with breast-conserving surgery and IMRT, the degree of desquamation seen was lower than in matched patients treated with conventional radiation: this difference was significant in a stepwise logistic regression model [42]. We are now using IMRT for patients requiring postmastectomy radiation in the setting of breast reconstruction; the hypothesis is that measures to decrease dose inhomogeneity within the reconstructed breast and skin with IMRT will reduce acute and long-term radiation complications and improve cosmetic outcome.

\section{Tumor control and complications with implant reconstruction and radiation}

A subpectoral placement should be performed to improve coverage of the implant, particularly when radiation therapy is anticipated $[24,43]$. This minimizes the risk of implant exposure either spontaneously during expansion or if there is desquamation of the skin during radiation. And since recurrences develop in the subcutaneous tissue or retained breast tissue anterior to the pectoralis fascia, submuscular placement also facilitates future surveillance of local recurrences because they are almost always clinically detectable and palpable anterior to the implant $[24,43]$. The local tumor control of patients with breast cancer treated by mastectomy has not been shown to be worse in those also undergoing implant reconstruction [24,43,44].

Complications after implant reconstruction and radiation may often be multifactorial in nature. It is often difficult to determine, but usually not clinically important, to what the degree a complication was due to radiation vs. the baseline risks from the reconstructive surgery or other adjuvant therapy. Some complications such as telangiectasias of the skin are clearly attributable only to radiation, but others such as infection may be caused by any number of factors. In patients treated with implant reconstruction without radiation, the baseline risk of serious complications has been reported to be relatively high, complication rates of approximately $10-40 \%$, including a significant percentage that are severe enough to require implant removal $[13,15,18,21,28]$. However, most comparative studies do indicate that the risk of complications is greater for irradiated patients [12-18,28]. The level of evidence of these studies is limited somewhat by their single institution and retrospective nature, and most are subject to potential biases or differences in patient or tumor characteristics between radiated and unirradiated patients. In addition, many studies are difficult to interpret because of a mix in patient groups 
between those with immediate or delayed reconstruction and pre- or post-implant radiation $[13,15]$.

Acute infection is a potentially serious early complication that may lead to implant failure in those having immediate pre-radiation reconstruction. Sandelin et al. [43] reported postoperative infections within 1 month in only $3 \%$ of patients after immediate implant reconstruction. Cordeiro et al. [28] reported a rate of only $1 \%$ in 542 patients after immediate reconstruction by tissue expander/implant without radiation. In many series with longer follow-up, the risk of infection for patients with implants and subsequent radiation is similarly low in the range of $5 \%$ or less $[21,25,28,45]$. However, Krueger et al. [13] reported infectious complications in $37 \%$ (7 of 19) of women with implants treated with radiation, compared with $19 \%$ (12 of 62 ) treated without radiation, although this did not reach statistical significance $(P=0.13)$. This high rate of infection compared to other studies may be due to longer follow-up (some occurred up to 13 months after surgery), the mixed population of patients with immediate or delayed and pre- or post-radiation implants, and the tendency for multi-institutional studies (the Krueger study involved 12 hospitals across USA and Canada) to have worse results than single-institution studies. The treating radiation oncologist should have a low threshold for starting antibiotics for prolonged courses in cases where there are early signs of infection, or as prophylaxis in cases of desquamation of the skin during or after radiation.

Later complications that may be attributable to postmastectomy radiation include implant capsular contracture, fibrosis of the skin and soft tissue, pain, hyperpigmentation and telangiectasias of the skin, and rarely direct implant exposure through the skin. Complications with implants have not been reliably shown to be related to adjuvant chemotherapy, diabetes, or smoking history $[12,13,21,25]$. In one study, tamoxifen was significantly associated with reconstruction failure [13] but not in others [21,25].

Most studies of implant reconstruction and radiation have reported relatively high rates of capsular contracture and implant loss. Rosato and Dowden [18] reported a high contracture rate of $64 \%$ (7 of 11 ) in implant patients subsequently radiated, that was significantly greater than the $10 \%$ in a comparison nonirradiated control group. Jackson et al. [46] reported that $30 \%$ (3 of 10 ) patients having radiation of a tissue expander ultimately experienced loss of the implant. Victor et al. [26] reported 13 patients with implant reconstruction and radiation had a good/excellent cosmetic result in only 54\%. Spear and Onyewu [15] reported a complication rate of $48 \%$ in irradiated patients that required the replacement of the implant or major revision by addition of a muscle flap for coverage. It should be noted that half of the patients had received radiation during their expansion in this series. Krueger et al. [13] reported a series of implant patients treated with radiation but with a mix of cases in timing between surgery and reconstruction and reconstruction and radiation. There was a $68 \%$ complication rate and $37 \%$ implant failure rate in 19 patients having expander/implant and radiation. A control group of 62 nonirradiated implant patients had a high $31 \%$ complication rate and $8 \%$ implant failure rate, but this was still lower than the irradiated patients. Chawla et al. [47] reported a 53\% rate of complications within 2 years of radiation therapy, and a good or excellent cosmesis in only $39 \%$ of 18 patients treated by expander/implant and radiation. Tallet et al. [21] reported 47 patients undergoing immediate subpectoral expander placement, radiation therapy, and then exchange for a permanent saline implant 4-6 months after radiation. There was a complication rate of $49 \%$, with $26 \%$ experiencing failure of the expander. Cosmesis was good or excellent in $54 \%$.

Two recent studies have shown more promising cosmetic results and low rates of serious complications and implant failure with immediate reconstruction using tissue expander/implant reconstruction. Anderson et al. [25] reported a large series of patients treated at Fox Chase Cancer Center with reconstruction and radiation. In this series, most patients underwent a delayed 'sandwich' technique of radiation of the fully infused tissue expander, followed by completion of reconstruction with the permanent saline implant after radiation. The 3-year complications in 50 patients with an implant reconstruction were $19 \%$. Of these, only $5 \%$ were considered serious: two patients required removal of their implant. The other $14 \%$ of complications were considered minor in four patients: two infections and two contractures. There was a major complication in $17 \%$ ( 1 of 6 ) patients receiving radiation before reconstruction, compared to $2 \%$ (1 of 44) patients receiving radiation to the tissue expander. Minor complications of infection or contracture were reported in 0 of 6 with radiation first and $9 \%$ (4 of 44) patients with tissue expansion first. The cosmesis was good or excellent in $82 \%$ of the implant patients. Cordeiro et al. [28] reported a study of 81 patients at Memorial Sloan-Kettering Cancer Center treated with immediate tissue expander/implant reconstruction and radiation. They were compared with a nonirradiated control group from the same study period. This series is the largest in the literature and the most homogenous - immediate expander placement at mastectomy, expansion 10-14 days after surgery, exchange to a permanent expander within 1 month of chemotherapy, and then radiation 1 month after the exchange. After a mean follow-up of almost 3 years, capsular contracture occurred in 
$68 \%$ of irradiated patients compared with $40 \%$ of unirradiated patients $(P=0.006)$, with Grade 3 or higher in $40 \%$ vs. $10 \%$. There was a statistically significant different rate of implant removal between irradiated $(11 \%)$ and unirradiated $(6 \%)$ patients. The cosmetic result was good or excellent in $80 \%$ of irradiated patients compared with $88 \%$ of nonirradiated patients $(P=0.004)$. Factors in common between these last two studies include a multidisciplinary team of physicians, immediate timing of implant reconstruction, completion of expansion prior to irradiation rather than concurrently, and uniform radiation therapy techniques. One area for future study with two-stage immediate reconstruction will be whether there are differences in outcomes due to timing the radiation to either a tissue expander or to the permanent implant.

\section{Conclusions}

This review has studied the implications of planned radiotherapy on breast reconstruction with implants. This challenge will become more common for the oncologic team treating breast cancer. This is due to many factors including surgical progress in mastectomy making it compatible with reconstruction, fading of past concerns about a negative impact of reconstruction on cancer recurrence and detection, and increasing evidence supporting postmastectomy radiation for a greater number of indications. A multidisciplinary approach to management is needed to carefully identify patients who may be appropriate candidates for implants, and to coordinate the necessary primary and adjuvant cancer therapies with the steps of the plastic surgical procedures. Immediate timing of reconstruction with tissue expander/implant provides the patient with important cosmetic and psychological benefits, while delayed reconstruction with implants also may be more difficult or impossible after chest wall irradiation. Experience with implant reconstruction and radiation in the past has been associated with relatively high rates of complications and/or implant loss compared with unirradiated patients. Advances in techniques of radiation that reduce dose heterogeneity including 3-D conformal radiation and most recently IMRT may reduce the acute and long-term side effects of radiation therapy in these patients. Recent studies of radiation therapy in the setting of immediate reconstruction now show the promise of low complication rates and good or excellent cosmetic results for most patients in the future.

\section{References}

1. Early Breast Cancer Trialists' Collaborative Group. Favourable and unfavorable effects on long-term survival of radiotherapy for early breast cancer: an overview of the randomised trials. Lancet 2000; 355: 1757-1770.

2. Fowble B. Postmastectomy radiation: then and now. Oncology (Huntington) 1997; 11: 213-239.

3. Cuzick J, Stewart H, Rutqvist L, et al. Cause-specific mortality in long-term survivors of breast cancer who participated in trials of radiotherapy. J Clin Oncol 1994; 12: 447-453.

4. Overgaard M, Hansen PS, Overgaard J, et al. Postoperative radiotherapy in high-risk premenopausal women with breast cancer who receive adjuvant chemotherapy. N Engl J Med 1997; 337: 949-955.

5. Overgaard M, Jensen M, Overgaard J, et al. Postoperative radiotherapy in high-risk postmenopausal breast-cancer patients given adjuvant tamoxifen: Danish Breast Cancer Cooperative Group DBCG 82c randomised trial. Lancet 1999; 353: 1641-1648.

6. Ragaz J, Olivotto IA, Spinelli JJ, et al. Locoregional radiation therapy in patients with high-risk breast cancer receiving adjuvant chemotherapy: 20 -year results of the British Columbia randomized trial. J Natl Cancer Inst 2005; 97: 116-126.

7. Taghian A, Jeong J, Mamounas E, et al. Patterns of locoregional failure in patients with operable breast cancer treated by mastectomy and adjuvant chemotherapy with or without tamoxifen and without radiotherapy: results from five national Surgical Adjuvant Breast and Bowel Project randomized clinical trials. J Clin Oncol 2004; 22: 4247-4254.

8. Wallgren A, Bonetti M, Gelber RD, et al. Risk factors for locoregional recurrence among breast cancer patients: results from International Breast Cancer Study Group trials I through VII. J Clin Oncol 2003; 21: 1205-1213.

9. National Comprehensive Cancer Network. NCCN practice guidelines for breast cancer. 2005: http://www.nccn.org/professionals/physician_gls/PDF/ breast.pdf (last accessed 28 July 2005).

10. Kronowitz SJ, Robb GL. Breast reconstruction with postmastectomy radiation therapy: current issues. Plast Reconstr Surg 2004; 114: 950-960.

11. Buchholz TA, Strom EA, Perkins GH, et al. Controversies regarding the use of radiation after mastectomy in breast cancer. Oncologist 2002; 7: 539-546.

12. Lin KY, Johns FR, Gibson J, et al. An outcome study of breast reconstruction: presurgical identification of risk factors for complications. Ann Surg Oncol 2001; 8: 586-591.

13. Krueger EA, Wilkins EG, Strawderman M, et al. Complications and patient satisfaction following expander/ implant breast reconstruction with and without radiotherapy. Int J Radiat Oncol Biol Phys 2001; 49: 713-721.

14. Vandeweyer E, Deraemaecker R. Radiation therapy after immediate breast reconstruction with implants. Plast Reconstr Surg 2000; 106: 56-58.

15. Spear SL, Onyewu C. Staged breast reconstruction with saline-filled implants in the irradiated breast: recent trends and therapeutic implications. Plast Reconstr Surg 2000; 105: 930-942.

16. Evans GR, Schusterman MA, Kroll SS, et al. Reconstruction and the radiated breast: is there a role for implants? Plast Reconstr Surg 1994; 96: 1111-1115.

17. Kraemer O, Andersen M, Siim E. Breast reconstruction and tissue expansion in irradiated versus not irradiated women after mastectomy. Scand J Plast Reconstr Surg Hand Surg 1996; 30: 201-206. 
18. Rosato RM, Dowden RV. Radiation therapy as a cause of capsular contracture. Ann Plast Surg 1994; 32: 342-345.

19. Dickson MG, Sharpe DT. The complications of tissue expansion in breast reconstruction: a review of 75 cases. Br J Plast Surg 1987; 40: 629-635.

20. Forman DL, Chiu J, Restifo RJ, et al. Breast reconstruction in previously irradiated patients using tissue expanders and implants: a potentially unfavorable result. Ann Plast Surg 1998; 40: 360-363.

21. Tallet AV, Salem N, Moutardier V, et al. Radiotherapy and immediate two-stage breast reconstruction with a tissue expander and implant: complications and esthetic results. Int J Radiat Oncol Biol Phys 2003; 57: 136-142.

22. Recht A, Gray R, Davidson NE, et al. Locoregional failure 10 years after mastectomy and adjuvant chemotherapy with or without tamoxifen without irradiation: experience of the Eastern Cooperative Oncology Group. J Clin Oncol 1999; 17: 1689-1700.

23. Freedman GM, Fowble BL, Hanlon AL, et al. A close or positive margin after mastectomy is not an indication for chest wall irradiation except in women aged fifty or younger. Int J Radiat Oncol Biol Phys 1998; 41: 559-605.

24. Hazard L, Miercort C, Gaffney D, et al. Local-regional radiation therapy after breast reconstruction: what is the appropriate target volume? Am J Clin Oncol 2004; 27: 555-564.

25. Anderson PR, Hanlon AL, Fowble BL, et al. Low complication rates are achievable after postmastectomy breast reconstruction and radiation therapy. Int $J$ Radiat Oncol Biol Phys 2004; 59: 1080-1087.

26. Victor SJ, Brown DM, Horwitz EM, et al. Treatment outcome with radiation therapy after breast augmentation or reconstruction in patients with primary breast carcinoma. Cancer 1998; 82: 1303-1309.

27. Schuster RH, Kuske RR, Young VL, et al. Breast reconstruction in women treated with radiation therapy for breast cancer: cosmesis, complications, and tumor control. Plast Reconstr Surg 1992; 90: 445-452.

28. Cordeiro PG, Pusic AL, Disa JJ, et al. Irradiation after immediate tissue expander/implant breast reconstruction: outcomes, complications, aesthetic results, and satisfaction among 156 patients. Plast Reconstr Surg 2004; 113: 877-881.

29. Freedman GM, Fowble BL, Hanlon AL, et al. Postmastectomy radiation and adjuvant systemic therapy: outcomes in high-risk women with stage II-III breast cancer and assessment of clinical, pathologic, and treatmentrelated factors influencing local-regional control. Breast J 1997; 3: 337-344.

30. Fowble B, Freedman G. Cancer of the breast. In: Wang CC (Ed.) Clinical Radiation Oncology: Indications, Techniques and Results, 2nd edition. New York: Wiley-Liss, Inc; 2000.

31. Intensity Modulated Radiation Therapy Collaborative Working Group. Intensity-modulated radiotherapy: current status and issues of interest. Int $J$ Radiat Oncol Biol Phys 2001; 51: 880-914.

32. Buchholz TA, Gurgoze E, Bice WS, et al. Dosimetric analysis of intact breast irradiation in off-axis planes. Int J Radiat Oncol Biol Phys 1997; 39: 261-267.
33. Das IJ, Cheng C, Fein DA, et al. Patterns of dose variability in radiation prescription of breast cancer. Radiother Oncol 1997; 44: 83-89.

34. Taylor ME, Perez CA, Halverson KJ, et al. Factors influencing cosmetic results after conservation therapy for breast cancer. Int J Radiat Oncol Biol Phys 1995; 31: 753-764.

35. Gorodetsky R, Lotan C, Piggot K, et al. Late effects of dose fractionation on the mechanical properties of breast skin following post-lumpectomy radiotherapy. Int J Radiat Oncol Biol Phys 1999; 45: 893-900.

36. Johansson S, Svensson H, Denekamp J. Dose response and latency for radiation-induced fibrosis, edema, and neuropathy in breast cancer patients. Int J Radiat Oncol Biol Phys 2002; 52: 1207-1219.

37. Hong L, Hunt M, Chui C, et al. Intensity-modulated tangential beam irradiation of the intact breast. Int $J$ Radiat Oncol Biol Phys 1999; 44: 1155-1164.

38. Kestin LL, Sharpe MB, Frazier RC, et al. Intensity modulation to improve dose uniformity with tangential breast radiotherapy: initial clinical experience. Int $J$ Radiat Oncol Biol Phys 2000; 48: 1559-1568.

39. Hurkmans CW, Cho BCJ, Damen E, et al. Reduction of cardiac and lung complication probabilities after breast irradiation using conformal radiotherapy with or without intensity modulation. Radiother Oncol 2002; 62: 163-171.

40. Vicini FA, Sharpe M, Kestin L, et al. Optimizing breast cancer treatment efficacy with intensity-modulated radiotherapy. Int J Radiat Oncol Biol Phys 2002; 54: 1336-1344.

41. Li J, Freedman G, Price R, et al. Clinical implementation of intensity-modulated tangential beam irradiation for breast cancer. Med Phys 2004; 31: 1023-1031.

42. Freedman GM, Anderson PR, Hanlon AL, et al. Intensity modulated radiation therapy (IMRT) decreases the acute skin toxicity for large-breasted women receiving radiation therapy for breast cancer. Int $J$ Radiat Oncol Biol Phys 2004; 60: S401-S402.

43. Sandelin K, Wickman M, Billgren A. Oncological outcome after immediate breast reconstruction for invasive breast cancer: a long-term study. Breast 2004; 13: 210-218.

44. Vandeweyer E, Hertens D, Nogaret JM, et al. Immediate breast reconstruction with saline-filled implants: no interference with the oncologic outcome? Plast Reconstr Surg 2001; 107: 1409-1412.

45. Nahabedian MY, Tsangaris T, Momen B, et al. Infectious complications following breast reconstruction with expanders and implants. Plast Reconstr Surg 2003; 112: 467-476.

46. Jackson WB, Goldson AL, Staud C. Postoperative irradiation following immediate breast reconstruction using a temporary tissue expander. J Natl Med Assoc 1994; 86: 538-542.

47. Chawla AK, Kachnic LA, Taghian AG, et al. Radiotherapy and breast reconstruction: complications and cosmesis with TRAM versus tissue expander/implant. Int $J$ Radiat Oncol Biol Phys 2002; 54: 520-526. 